山्山FRANÇAISE

$>\mathrm{DE}$

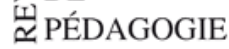

\section{Revue française de pédagogie}

Recherches en éducation

182 | 2013

Connaissances et politiques d'éducation : quelles interactions?

\title{
Doussot Sylvain. Didactique de l'histoire. Outils et pratiques de l'enquête historienne en classe
}

Rennes : Presses universitaires de Rennes, 2011, 316 p.

\section{Didier Cariou}

\section{OpenEdition \\ Journals}

\section{Édition électronique}

URL : http://journals.openedition.org/rfp/4046

DOI : $10.4000 / \mathrm{rfp} .4046$

ISSN : 2105-2913

\section{Éditeur}

ENS Éditions

\section{Édition imprimée}

Date de publication : 28 août 2013

Pagination : 147-149

ISSN : 0556-7807

Référence électronique

Didier Cariou, « Doussot Sylvain. Didactique de l'histoire. Outils et pratiques de l'enquête historienne en classe », Revue française de pédagogie [En ligne], 182 | 2013, mis en ligne le 28 août 2013, consulté le 22 septembre 2020. URL : http://journals.openedition.org/rfp/4046 ; DOI : https://doi.org/10.4000/rfp. 4046 
à supporter ». Ce ne sont pas les concepts ordinaires de la didactique, et la question se pose donc de la facilité/difficulté de la communication de l'équipe avec la communauté des chercheurs et celle des enseignants. Certains lecteurs seront surpris par une problématique à leurs yeux «étrange ». Et pourtant l'ouvrage est très intéressant : passée la surprise initiale provoquée chez le lecteur par les catégories utilisées, celui-ci s'intéressera aux propos tenus par les enseignants dont le témoignage est recueilli, et qui illustrent les attitudes et les représentations plus ou moins conscientes qui orientent la subjectivité des enseignants dans leur activité.

Le « déjà-là » désigne les filtres hérités de l'expérience passée, qui modifient et biaisent les objectifs de l'enseignant ainsi que son interprétation des observations qu'il peut faire dans sa classe. La comparaison entre un entretien avant le cours et un entretien après met assez aisément en évidence le décalage entre les objectifs institués et ceux de l'enseignant. En d'autres termes, l'enseignant n'est pas maître dans son institution.

Le terme « épreuve » désigne l'activité de l'enseignant en situation, avec ce qu'elle comporte d'ajustement à l'imprévu et d'écart éventuel avec l'attente de l'enseignant. On n'est pas éloigné cette fois de l'idée de contrat didactique ? L'aprèscoup permet de mettre cet écart en évidence.

Si l'enseignant est « supposé savoir », il ne peut qu'être surpris par les difficultés qu'il rencontre et par son embarras pour les surmonter, mais il lui est aussi « impossible de supporter » certaines faiblesses des élèves, par exemple leur faible engagement dans l'activité sportive que, justement, il privilégie lui-même en raison de son histoire personnelle.

Mettre ces différents phénomènes en évidence dans une variété d'activités sportives comme la gymnastique, la boxe française ou le volley-ball suppose évidemment que l'on tienne compte des spécificités de chaque activité, mais, dans tous les cas le « je » de l'enseignant est bel et bien un facteur important d'orientation de son activité et de son jugement. L'expertise professionnelle n'est pas l'expérience personnelle, les deux semblant plutôt s'infléchir l'une l'autre.

Plusieurs autres thèmes intéressent l'équipe de didactique clinique, comme l'évaluation ou encore l'introduction dans les programmes de contenus nouveaux, l'éducation à la santé par exemple.

Les préoccupations concernant la personne de l'enseignant commencent à être étudiées de plusieurs manières dans la recherche, en raison de la dualité des fonctions de l'activité de travail, à la fois une fonction productive (effets sur le monde extérieur) et une fonction constructive (effets sur la personne elle-même) ; aussi en raison du retour réflexif sur l'action, qui donne à l'expérience personnelle un véritatable statut conceptuel ; aussi en raison de la défense personnelle que doit assurer l'enseignant au cours de son interaction avec les élèves.

Les considérations méthodologiques occupent une bonne partie des contributions à l'ouvrage, notamment les considérations concernant le choix et la construction des cas individuels retenus pour l'analyse. La didactique clinique de l'EPS représente donc, à mon avis, un apport original à la didactique, et ajouterai-je, à la psychologie.

Gérard Vergnaud

Université Paris 8-Vincennes-Saint-Denis, laboratoire Paragraphe

DOUSSOT Sylvain. Didactique de l'histoire. Outils et pratiques de l'enquête historienne en classe. Rennes: Presses universitaires de Rennes, 2011, 316 p.

L'ouvrage de Sylvain Doussot, rédigé à partir de sa thèse soutenue en 2009, marque une importante avancée pour la didactique de l'histoire. Il fait notamment progresser le débat sur la question de la rupture et de la continuité entre l'histoire scolaire et l'histoire universitaire (Lautier \& Allieu-Mary, 2008).

Il montre que le travail en classe d'histoire ne saurait se réduire à un ensemble de techniques rencontrées dans les situations habituelles de classe, décrites suivant le modèle de la discipline scolaire (Chervel, 1998) qui perçoit l'histoire scolaire comme éloignée de sa discipline de référence. Certains didacticiens envisagent en effet la continuité entre les démarches de l'histoire universitaire et celles de l'histoire enseignée. Comment dès lors penser une dialectique de la continuité et de la rupture, comment envisager les conditions de l'apprentissage de la pensée historienne en classe, tout en tenant compte des contraintes de la forme scolaire (p. 51) ? À cet effet, S. Doussot propose des pistes pour penser et travailler la référence savante en classe d'histoire.

Ce travail s'intègre dans le cadre théorique de la problématisation élaboré par M. Fabre (2009). Il ne s'agirait plus d'envisager les savoirs comme le résultat de la résolution d'un problème construit par ailleurs et selon une dimension strictement propositionnelle du savoir (savoir que...). Il s'agirait plutôt d'envisager les savoirs comme étant liés à des problèmes et faisant état de nécessités (savoir qu'il ne saurait en être autrement). Ce questionnement engagerait un processus de problématisation analogue à celui des historiens quand il permet d'explorer les différents possibles qui expliquent une situation historique. Les élèves produiraient alors des hypothèses non seulement pour comprendre comment les choses se sont passées mais surtout pourquoi elles se sont passées ainsi et pas autrement (Koselleck, 1997, 
p. 217 et p. 229). De cette manière, la Révolution française n'est plus un évènement en soi dont il convient de connaître les principaux faits et personnages, sa connaissance devient - ou redevient - la réponse à des questions d'historiens qui en proposent des découpages temporels et des interprétations. On sortirait ainsi de l'aporie de la transposition didactique qui produit la réification des savoirs scolaires.

À cet effet, S. Doussot analyse les échanges en classe, les documents utilisés et les productions d'élèves lors de trois séquences menées en classe de CM1 (la seigneurie au Moyen Âge), de quatrième (la chute de la monarchie constitutionnelle en 1792) et de troisième (la Guerre froide). Il décrit notamment l'usage par les élèves de listes et de tableaux, envisagé sous l'angle de l'instrumentation de pratiques qui ont à voir avec celles des historiens. L'objectif n'est pas seulement de conduire les élèves à prélever des informations dans des documents pour remplir des cellules de tableaux - comme l'exige la forme scolaire de l'histoire - mais à s'emparer de ces outils pour lister et classer des informations, pour faire jouer les données selon leur disposition spatiale afin d'en proposer des explications. Comme les cellules du tableau juxtaposent des éléments disjoints, il appartient aux élèves de restituer les contraintes et les contingences qui, par exemple, relient implicitement la fuite à Varennes, la déclaration de guerre et le manifeste de Brunswick, afin de proposer une explication de la chute de la monarchie (p. 202). La dimension bi-dimensionnelle de ces outils graphiques, disposant spatialement les données et les contraintes, matérialise un espace-problème pour explorer un problème historique (p. 23). Les élèves ne suivent pas un trajet qui les mènerait du faux au vrai par une recherche de la «bonne » réponse, ils réalisent un parcours qui leur permet de déterminer ce qui est possible et ce qui ne l'est pas, en examinant les différentes configurations historiographiques (Prost \& Winter, 2004) du problème, en convoquant les données recueillies et les interprétations antérieures déjà validées par la classe. Conformément à la théorie anthropologique de l'écriture (Goody, 1979), à la théorie de la genèse instrumentale (Rabardel, 1999) et à l'approche socio-historique du langage (Jaubert, 2007), les savoirs ne sauraient donc se poser indépendamment des pratiques de leur mise en texte par les élèves (p. 95). Dans une étude de cas déjà publiée dans cette revue (Doussot, 2010), il apparaît que la continuité linéaire entre la fuite du roi à Varennes en juin 1791 et sa chute le 10 août 1792 n'explique rien car le problème est d'abord le suivant : pourquoi les Parisiens ont-ils mis fin à la royauté en août 1792 et pas en juin 1791 ? Il devient nécessaire de travailler sur ce que les Parisiens savaient et pensaient des intentions du roi au fil des évènements pour construire le registre des contraintes et des explications de l'évènement. La disposition spatiale des données dans un tableau - qui intègre la déclaration de guerre et le manifeste de Brunswick dans cette succession temporelle - fait apparaître que les Parisiens ne prirent conscience de la trahison du roi qu'à l'été 1792 , avec la publication du manifeste de Brunswick (p. 115).

En lien avec l'épistémologie bachelardienne de la problématisation, les outils graphiques apparaissent comme les instruments de la rupture (à ne pas confondre avec la coupure car elle n'exclut pas la continuité) entre le sens commun et la science. S. Doussot propose ainsi une réponse aux débats qui agitent la communauté des didacticiens de l'histoire en montrant que la rupture ne se situe pas entre les élèves et les historiens, mais entre les savoirs quotidiens mobilisés spontanément par les élèves et les savoirs des historiens (p. 54). En effet, les représentations initiales des élèves peuvent aussi bien constituer un appui qu'un obstacle à la construction du savoir. Instrumenter les pratiques des élèves avec des listes et des tableaux conduit ces derniers à parcourir un espace-problème pour explorer d'autres possibles (le roi peut trahir ses sujets pour des raisons politiques et des intérêts personnels) et engager éventuellement une rupture avec les représentations et le sens commun (le roi est nécessairement le protecteur de ses sujets). L'obstacle épistémologique au sens de Bachelard ne consisterait pas en un savoir trop éloigné des élèves et masqué par leurs représentations premières. Il se tiendrait au cœur du savoir lui-même. Il peut être surmonté par l'émission de nouvelles hypothèses instrumentées par des outils graphiques. En tant que « technologie de l'intellect » (Goody, 1979), ces derniers sont des instruments de la secondarisation du savoir qui se tient également au cœur du « modèle intermédiaire d'apprentissage de l'histoire » (Lautier, 1997).

Au total, S. Doussot considère que, pour rapprocher les pratiques scolaires des pratiques historiennes, il est nécessaire d'instituer la classe en une " communauté historienne scolaire » (Bernié, 2002), en un espace d'échanges et de controverses où se construiraient l'enquête et les pratiques textuelles des élèves (p. 281). Il propose d'orienter l'enseignement de l'histoire selon une logique post-moderne qui met l'accent sur la négociation dans la classe de la signification des différentes versions du passé (p. 260-262). L'enseignement de cette histoire problématisée opérerait ainsi la médiation entre la communauté des historiens et la communauté historienne scolaire qu'il souhaite faire advenir dans sa classe.

Ces propositions, appuyées sur un matériau empirique d'une très grande richesse et armées par un cadre théorique rigoureux, semblent d'une grande portée. Elles ouvrent de nombreuses pistes de recherche sur la nature du savoir historique, sur le renouvellement possible de la forme scolaire, sur l'articulation de l'histoire enseignée à l'historiographie et à l'épistémologie de l'histoire. De notre point de vue, elles 
pourraient ouvrir au moins deux types de questionnement. Ce cadre - nécessairement « contraint » afin d'introduire les élèves à une démarche de problématisation - laisse en suspens la question de l'effectivité des apprentissages dans les situations habituelles de classe. Il pose également la question des croisements possibles ou impossibles entre la théorie de la problématisation et des autres cadres théoriques mobilisés en didactique de l'histoire.

Didier Cariou

Université de Bretagne occidentale, CREAD EA 3875

\section{BIBLIOGRAPHIE}

BERNIÉ J.-P. (2002). « L'approche des pratiques langagières scolaires à travers la notion de "communauté discursive" : un apport à la didactique comparée ? ". Revue française de pédagogie, no 141, p. 77-88.

CHERVEL A. (1998). La culture scolaire. Une approche historique. Paris : Belin.

DOUSSOT S. (2010). «Pratiques de savoir en classe et chez les historiens : une étude de cas au collège ". Revue française de pédagogie, no 173, p. 85-104.

FABRE M. (2009). Philosophie et pédagogie du problème. Paris : Vrin.

GOODY J. (1979). La raison graphique. La domestication de la pensée sauvage. Paris : Éd. de Minuit.

JAUBERT M. (2007). Langage et construction de connaissances à l'école. Un exemple en sciences. Bordeaux : Presses universitaires de Bordeaux.

KOSELLECK R. (1997). L'expérience de l'histoire. Paris : Gallimard ; Seuil.

LAUTIER N. (1997). À la rencontre de l'histoire. Villeneuve d'Ascq : Presses universitaires du Septentrion.

LAUTIER N. \& ALLIEU-MARY N. (2008). « La didactique de l'histoire ". Revue française de pédagogie, no 162, p. 95-131.

PROST A. \& WINTER J. (2004). Penser la Grande Guerre. Un essai d'historiographie. Paris : Seuil.

RABARDEL P. (1999). « Le langage comme instrument ? Éléments pour une théorie instrumentale élargie ». In $\mathrm{Y}$. Clot (dir.), Avec Vygotski. Paris : La Dispute.

MARGOLINAS Claire \& WOZNIAK Floriane. Le nombre à l'école maternelle, une approche didactique. Bruxelles : De Boeck, 2012, 130 p.

Cet ouvrage s'adresse bien sûr aux formateurs mais aussi bien, au vu du format réduit et du nombre normalisé de pages, aux professeurs désirant soit réfléchir aux équilibres professionnels qu'ils ont trouvés, soit argumenter de leurs bonnes pratiques, et encore aux chercheurs désireux d'envisager l'ensemble des problèmes rencontrés par un professeur et faire l'état de la question.

Sorti en 2012, l'ouvrage est le huitième de la ligne «Pédagogie » ouverte par De Boeck dans la collection « Le point sur... ». Il suit Faire la classe à l'école maternelle, Savoir enseigner dans le secondaire. Didactique générale, Pédagogie différenciée, La régulation des apprentissages en classe, Fabriquer le savoir enseigné, Enseigner les sciences. Problèmes, débats et savoirs scientifiques en classe, Inclusion scolaire. Dispositifs et pratiques pédagogiques, etc. À l'évidence, les responsables de collection, Sabine Kahn et Bernard Rey, cherchent l'équilibre entre pédagogie et didactique, manières d'enseigner et contenus d'enseignement. C'est un choix qui nous paraîtrait quasi impossible en France (Mercier, 2010), mais on sait qu'en Belgique comme en Allemagne les deux dimensions pédagogique et didactique sont liées dans toute pensée sur l'éducation visant l'intelligibilité des phénomènes, sinon la scientificité de leur approche, et recouvrent pratiquement ce qu'en France on nomme sciences de l'éducation. Cette unité conduit tout auteur à penser, ne serait-ce qu'implicitement, la place de l'autre approche.

On peut voir la différence avec notre situation nationale dans le fait que c'est un psychologue, Michel Fayol (2012), qui au même moment publie dans la collection «Que saisje » 128 pages intitulées L'acquisition du nombre, un ouvrage donc le public est proche de celui de la collection « Le point sur... » mais qui, au nom d'une psychologie expérimentale de laboratoire pour qui le nombre est un concept, fait peu de place à ce que l'on pourrait apprendre d'un travail en classe et de l'observation d'un collectif d'élèves, et qui ne donne pas d'analyse épistémologique de l'objet mathématique que pourrait être «le nombre » et des pratiques qui lui sont rattachées dans notre culture.

\section{Enseigner les nombres en maternelle ?}

La question d'un enseignement numérique en maternelle s'est imposée peu à peu comme problème, dans la société ; l'école, au fur et à mesure de l'intégration de la grande section (dernière année de maternelle) dans le cycle des apprentissages fondamentaux qui se continue en $\mathrm{CP}$ (première primaire) et CE1 (deuxième primaire), a envisagé de prendre en charge les éléments premiers de cet enseignement. Cette continuité déclarée a conduit les professeurs à anticiper sur les premiers enseignements disciplinaires, lecture et écriture, mais aussi mathématiques ou français, éducation physique ou sciences : ce livre vient donc à point pour donner des éléments de réponse aux questions que peuvent alors se poser les professeurs et leurs formateurs. 